\title{
Association between CYP17 T-34C rs743572 and breast cancer risk
}

\author{
Jing Sun ${ }^{1, *}$, Hong Zhang ${ }^{2, *}$, Meiyan Gao ${ }^{3, *}$, Zhishu Tang ${ }^{1}$, Dongyan Guo ${ }^{4}$, Xiaofei \\ Zhang ${ }^{4}$, Zhu Wang ${ }^{5}$, Ruiping $\mathrm{Li}^{5}$, Yan Liu ${ }^{5}$, Wansen Sun ${ }^{5}$ and Xi Sun ${ }^{6}$ \\ ${ }^{1}$ Department of Shaanxi Collaborative Innovation Center of Chinese Medicinal Resources Industrialization, Shaanxi University \\ of Chinese Medicine, Xianyang, Shaanxi, China \\ ${ }^{2}$ Department of Neurology, Second Affiliated Hospital, School of Medicine, Xi'an Jiaotong University, Xi'an, Shaanxi, China \\ ${ }^{3}$ Clinical Laboratory, Shaanxi Provincial Hospital of traditional Chinese medicine, Xi'an, Shaanxi, China \\ ${ }^{4}$ College of Pharmacy, Shaanxi University of Chinese Medicine, Xianyang, Shaanxi, China \\ ${ }^{5}$ Department of Integrated Traditional Chinese and Western Medicine, Second Affiliated Hospital, School of Medicine, Xi'an \\ Jiaotong University, Xi'an, Shaanxi, China \\ ${ }^{6}$ Department of General Medicine, Second Affiliated Hospital, School of Medicine, Xi'an Jiaotong University, Xi'an, Shaanxi, \\ China \\ *These authors contributed equally to this work
}

Correspondence to: Wansen Sun, email: sunwansen41@163.com Xi Sun, email: sunxi132@163.com

Keywords: breast cancer; rs743572; polymorphism

Received: September 04, $2017 \quad$ Accepted: December 18, $2017 \quad$ Published: December 26, 2017

Copyright: Sun et al. This is an open-access article distributed under the terms of the Creative Commons Attribution License 3.0 (CC BY 3.0), which permits unrestricted use, distribution, and reproduction in any medium, provided the original author and source are credited.

\section{ABSTRACT}

Association between CYP17 T-34C (rs743572) polymorphism and breast cancer (BC) risk was controversial. In order to derive a more definitive conclusion, we performed this meta-analysis. We searched in the databases of PubMed, EMBASE and Cochrane for eligible publications. Pooled odds ratios (ORs) with $95 \%$ confidence intervals (95\% CIs) were used to assess the strength of association between CYP17 T-34C polymorphism and breast cancer risk. Forty-nine studies involving 2,7104 cases and 3,4218 control subjects were included in this meta-analysis. In overall, no significant association between CYP17 T-34C polymorphism and breast cancer susceptibility was found among general populations. In the stratified analysis by ethnicity and source, significant associations were still not detected in all genetic models; besides, limiting the analysis to studies with controls in agreement with HWE, we also observed no association between CYP17 T-34C polymorphism and breast cancer risk. For premenopausal women, we didn't detect an association between rs743572 and breast cancer risk; however, among postmenopausal women, we observed that the association was statistically significant under the allele contrast genetic model $(O R=1.10,95 \% C I=1.03-1.17, P=0.003)$, but not in other four models. In conclusion, rs743572 may increase breast cancer risk in postmenopausal individuals, but not in premenopausal folks and general populations.

\section{INTRODUCTION}

Breast cancer $(\mathrm{BC})$, the most frequent malignant neoplasm among female worldwide, accounts for approximate $25 \%$ of women malignant tumor. It is reported that 1.67 million people were diagnosed as $\mathrm{BC}$ ever year, therefore it has become a serious health issue, especially in the developing countries [1]. It is well known that the lifetime presence of the estrogen in the blood is an important pathogenic factor of $\mathrm{BC}$, and this is in consistence with the low incidence of the breast cancer in males that is due to the lower estrogen levels and lower breast tissue volume. By now, researches on the status of hormone receptors and/or menopause associated with 
genetic alterations in $\mathrm{BC}$ risk have attracted an increasing number of attention, and lots of genes, including BRIPI, CHEK2, MDM, TGFB, TP53, BRCA1, BRCA2, and PTEN, and also several gene polymorphisms. Among genes of this family, CYP17, CYP19 and CYP1A1 have important functions in synthesis, metabolism and maintaining the levels of the androgen and estrogen hormones [2]. Previous published reasearches have demonstrated that estrogen act as a crucial role in the formation of $\mathrm{BC}$; in addition, evidences have also been found about the positive role of cell surface receptors of estrogen in tumorigenesis [3]. Nevertheless, the precise mechanism behind estrogen in the formation of $\mathrm{BC}$ remains unknown. Previous studies have indicated that cytochrome P450c17 $\alpha$, which is a key enzyme in the synthesis of estrogen, and could increase the breast neoplasm risk [4]. The cytochrome P450c17 $\alpha$ enzyme, predominantly catalyzes the formation of the precursor dehydroepiandrosterone (DHEA). Meanwhile, precursor DHEA could further be converted into estrogen through a succession of tissue-specific pathways $[5,6]$. Estrogen, plays a vital part in the etiology of BC and identified the risk between estrogen and $\mathrm{BC}$ could well elucidate the biosynthesis and metabolism mechanisms. So far, more and more researches have demonstrated the correlation of estrogen-related genes genetic variations with $\mathrm{BC}$ risk. The CYP17T-34C (rs743572) polymorphism which is located on the human chromosome 10, in the 50-untranslated region has been most commonly reported [7].

Many studies about the genetic mutations or SNP occurring in CYP17 gene could enhance CYP17's transcription rate and increase the enzyme cytochrome P450c17 level, resuling in an increasing number of bioavailable estrogen, which is likely to affect the risk and aggressiveness of BC [8]. But many previous article results between rs743572 mutations and $\mathrm{BC}$ risk remain conflicting: Han's research [9] revealed that no statistically meaningful correlation of rs743572 with risk of BC. However, significant correlation was found between rs743572 and BC risk in another research on the same theme [10]. Since few new high-quality investigations were published, we performed this study to take a more precise evaluation of rs 743572 with the risk of BC.

\section{RESULTS}

\section{The main feature of included studies}

As showed in Figure 1, 331 references were retrieved at first based on our selection strategy. 186 papers were remained after removing the duplicate reports. After reading titles and abstracts, we excluded 104 studies which were clearly unrelated. In the end, the whole of the rest of the papers were checked based on the inclusion and exclusion criteria. Finally, forty-nine studies on rs743572 and the risk of $\mathrm{BC}$ were eventually included in our study.
Thirteen articles showed the number of three genotypes (TT, TC, and CC) among premenopausal women, and thirteen studies report $\mathrm{TT}, \mathrm{TC}$, and $\mathrm{CC}$ number in postmenopausal women. Main information of included studies were shown in Table 1. Among these qualified researches, seventeen were performed in Asians, twentyfive in Caucasians, one in Africans, one in both Asians and Caucasians, one in both Africans and Caucasians, and four in mixed ethnicity. Moreover, twenty-two studies were considered as moderate-quality studies (NOS scores of these researches were 4-6), and other twenty-seven studies were considered as high-quality studies (NOS scores of these studies were seven or above). Except for four included researches were not in agreement with HardyWeinberg equilibrium (HWE), genotype distributions in the control groups of other 45 researches were all satisfied with HWE.

\section{Meta-analysis results}

Meta-analysis results among overall populations, distribution of this polymorphism in case groups and control groups are presented in Table 2. For premenopausal women and postmenopausal women, distribution of this polymorphism in case groups and control groups are presented in Table 3, and the main outcome of our study are shown in Tables 4 and 5 .

In overall populations, the association of CYP17 T-34C polymorphism with BC susceptibility was studied in forty-nine researches including 27,104 cases and 34,218 controls. No significant correlation was found between this polymorphism and BC susceptibility among any of the five genetic models: $\mathrm{T} / \mathrm{C}(\mathrm{OR}=0.99,95 \%$ $\mathrm{CI}=0.96-1.01, P=0.281), \mathrm{TT} / \mathrm{CC}(\mathrm{OR}=0.99,95 \%$ $\mathrm{CI}=0.98-1.01, P=0.309), \mathrm{TC} / \mathrm{CC}(\mathrm{OR}=0.98,95 \%$ $\mathrm{CI}=0.93-1.03, P=0.365), \mathrm{TT}+\mathrm{TC} / \mathrm{CC}(\mathrm{OR}=0.98,95 \%$ $\mathrm{CI}=0.93-1.02, P=0.287)$ and TT/TC $+\mathrm{CC}(\mathrm{OR}=0.99$, $95 \% \mathrm{CI}=0.95-1.02, P=0.463)$. Analogously, further subgroup analysis by ethnicity and source found similar results that in all the ethnic groups, $\mathrm{HB}$ group and $\mathrm{PB}$ group there is no significant correlation between rs 743572 and BC susceptibility. Moreover, if we only analyze the studies with controls in agreement with HWE, no correlation between rs743572 and BC risk were observed (Table 4) (Figure 2).

In premenopausal individuals, thirteen included researches with 2, 029 breast cancer case groups and 2, 920 control groups were eventually included. There is no statistical correlation of rs743572 with breast cancer susceptibility in T/C model, the TT/CC, the TC/CC, the $\mathrm{TT}+\mathrm{TC} / \mathrm{CC}$, and the TT/TC+CC $(\mathrm{OR}=1.02$ with $95 \% \mathrm{CI}$ $0.93-1.10, \mathrm{OR}=1.01$ with $95 \%$ CI $0.85-1.20, \mathrm{OR}=0.97$ with $95 \%$ CI $0.83-1.14, \mathrm{OR}=0.95$ with $95 \%$ CI $0.81-1.10$, and $\mathrm{OR}=1.04$ with $95 \%$ CI $0.92-1.18$, respectively). In postmenopausal women, significant correlation was found in $\mathrm{T} / \mathrm{C}$ model $(\mathrm{OR}=1.10,95 \% \mathrm{CI}=1.03-1.17, P=0.003)$ 
(Table 5) (Figure 3). However, there were no significant associations between the rs743572 polymorphism and breast cancer risk in other genotype distributions: TT/ $\mathrm{CC}(\mathrm{OR}=0.96,95 \% \mathrm{CI}=0.84-1.10, P=0.539), \mathrm{TC} / \mathrm{CC}$ $(\mathrm{OR}=0.96,95 \% \mathrm{CI}=0.85-1.08, P=0.478), \mathrm{TT}+\mathrm{TC} / \mathrm{CC}$ $(\mathrm{OR}=0.96,95 \% \mathrm{CI}=0.85-1.08, P=0.930)$ and TT/TC $+\mathrm{CC}$ $(\mathrm{OR}=0.99,95 \% \mathrm{CI}=0.90-1.08, P=0.357)$ (Table 5).

\section{Sensitivity analysis}

Even though four researches included in our studies were not conformed to the HWE balance $(P<0.05)$, final consequences were not changed when we excluded the abovementioned four studies. Besides, after performing the sensitivity analysis, the pooled OR values were not statistically significant changed when we delete each of the researches, indicating that this study has good stability and reliability.

\section{Heterogeneity analysis}

Heterogeneity was obtained by $\mathrm{Q}$ statistic. When the $P$ value more than 0.1 in the $Q$ test, then the fixedeffect models were selected to conduct relevant statistical analysis; otherwise, random-effect models were selected.

\section{Publication bias}

No statistical evidence of publication bias was found in the Begg's test and Egger's test. What's more, funnel plot also did not show any evidence of obvious asymmetry (Table 4) (Figure 4).

\section{DISCUSSION}

With the popularization and the rapid development of technology in the field of medicine, people have a deeper recognition of breast cancer. However, the specific mechanisms of the occurrence and the development of this cancer remain unclear. It is well established that estrogen involves in the development of mammary gland and plays crucial role in initiating of $\mathrm{BC}$ [2]. Extensive evidences have also been demonstrated that lifetime exposure to endogenous and/or exogenous estrogen, increased the risk of the morbidity of breast cancer [11]. Besides, estrogen plays a positive role of cell surface receptors of in tumorigenesis $[2,3]$. Significance of genes functioning in steroid hormone synthesis is well established in breast cancer susceptibility. CYP17, a commonly known gene could code for the cytochrome P450c17 $\alpha$ enzyme that is one of the key enzymes participated in estrogen biosynthesis [4]. CYP17 T-34C polymorphism, in the region (5'-UTR) of CYP17, has been reported up-regulate CYP17 transcription in some studies but not in others [7]. The functional impact of the $\mathrm{T} / \mathrm{C}$ change is still an unresolved mystery. Moreover several studies have reported conflicting results with respect to menopausal status and CYP17 polymorphism. Hence, for the purpose of acquire a more accurate assessment of the association

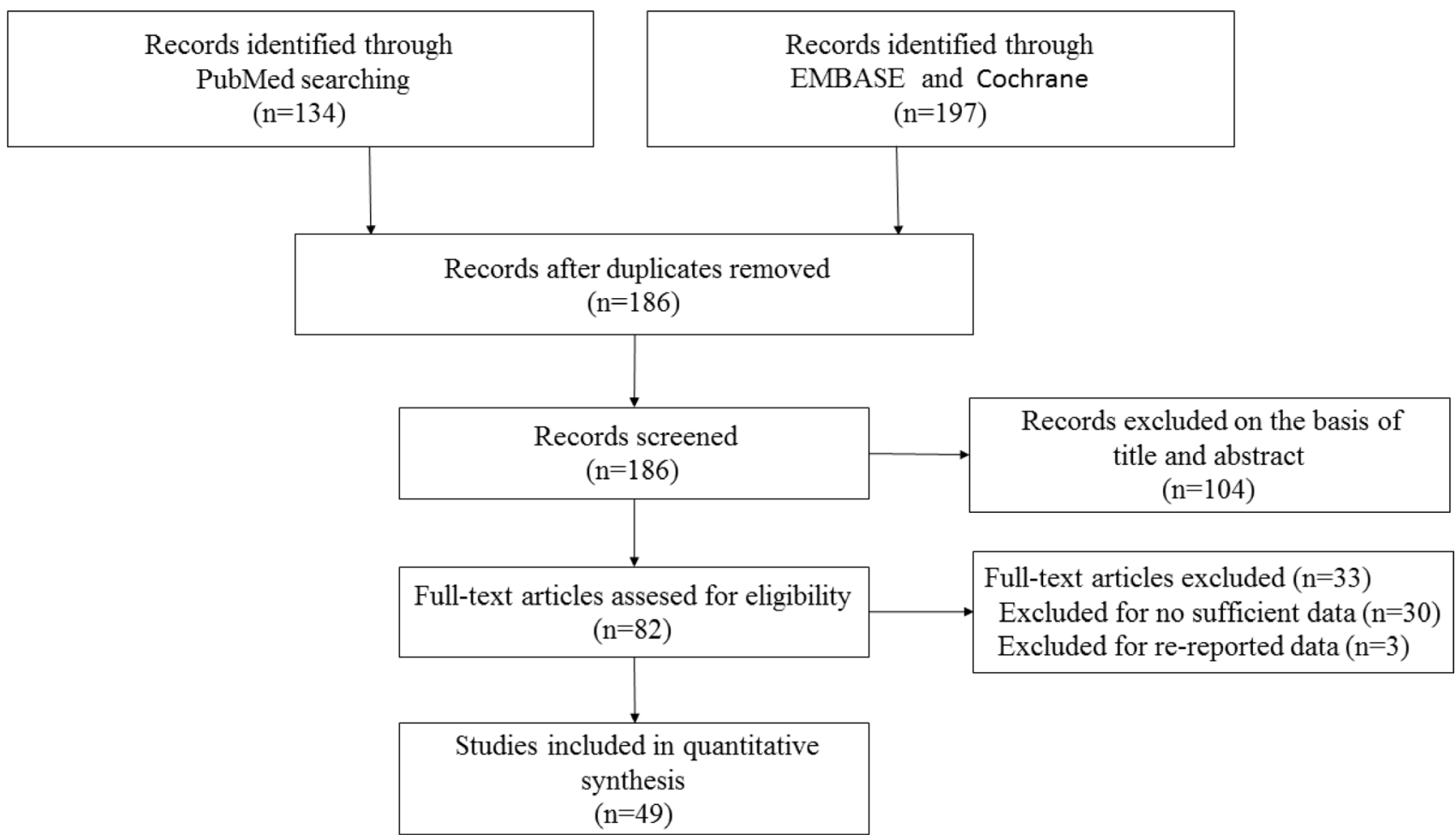

Figure 1: Flow diagram of the selection of the studies in this meta-analysis. 
Table 1: Characteristics of studies included in the meta-analysis

\begin{tabular}{|c|c|c|c|c|c|c|c|}
\hline First author & Year & Country & Ethnicity & $\begin{array}{c}\text { Source } \\
\text { of control }\end{array}$ & $\begin{array}{c}\text { Number } \\
\text { (case/control) }\end{array}$ & $\begin{array}{c}\text { HWE } \\
(P \text { value })\end{array}$ & NOS \\
\hline Dunning [17] & 1998 & UK & Caucasian & PB & $835 / 591$ & 0.261 & 7 \\
\hline Weston [21] & 1998 & USA & Caucasian & $\mathrm{HB}$ & $103 / 205$ & 0.449 & 6 \\
\hline Weston [21] & 1998 & USA & African & HB & $20 / 35$ & 0.253 & 6 \\
\hline Helzlsouer [22] & 1998 & USA & Caucasian & PB & $109 / 113$ & 0.549 & 6 \\
\hline Bergman [23] & 1999 & Sweden & Caucasian & PB & $109 / 117$ & 0.304 & 6 \\
\hline Haiman [24] & 1999 & USA & Caucasian & PB & $436 / 618$ & 0.391 & 7 \\
\hline Huang [25] & 1999 & China & Asian & PB & $123 / 126$ & 0.972 & 6 \\
\hline Young [26] & 1999 & UK & Caucasian & PB & $39 / 58$ & 0.732 & 5 \\
\hline Kristensen [27] & 1999 & Norway & Caucasian & PB & $510 / 201$ & 0.351 & 7 \\
\hline Hamajima [28] & 2000 & Japan & Asian & $\mathrm{HB}$ & $144 / 166$ & 0.044 & 6 \\
\hline Kuligina [29] & 2000 & Russia & Caucasian & HB & $240 / 182$ & 0.017 & 6 \\
\hline Mitrunen [30] & 2000 & Finland & Caucasian & PB & $479 / 480$ & 0.967 & 7 \\
\hline Feigelson [18] & 2001 & USA & Mixed & PB & $850 / 1508$ & 0.335 & 7 \\
\hline Gudmundsdottir [31] & 2003 & Iceland & Caucasian & PB & $500 / 395$ & 0.131 & 7 \\
\hline $\mathrm{Wu}[32]$ & 2003 & Singapore & Asian & PB & $188 / 671$ & 0.512 & 6 \\
\hline Ambrosone [33] & 2003 & USA & Caucasian & PB & $207 / 188$ & 0.130 & 7 \\
\hline Tan [34] & 2003 & China & Asian & PB & $250 / 250$ & 0.117 & 7 \\
\hline Hefler [35] & 2004 & Austria & Asian & PB & $388 / 1698$ & 0.455 & 7 \\
\hline Ahsan [36] & 2004 & USA & Mixed & HB & $313 / 271$ & 0.457 & 6 \\
\hline Chacko [37] & 2005 & India & Asian & $\mathrm{HB}$ & $140 / 140$ & 0.133 & 6 \\
\hline Einarsdo'ttir [38] & 2005 & Sweden & Caucasian & PB & $1499 / 1338$ & 0.885 & 7 \\
\hline Shin [39] & 2005 & Korean & Asian & HB & $462 / 337$ & 0.134 & 7 \\
\hline Verla-Tebit [40] & 2005 & Germany & Caucasian & PB & $527 / 904$ & 0.380 & 7 \\
\hline Hopper [41] & 2005 & Australia & Caucasian & PB & $1404 / 788$ & 0.697 & 7 \\
\hline Onland-More [42] & 2005 & Netherlands & Caucasian & $\mathrm{PB}$ & $335 / 373$ & 0.189 & 7 \\
\hline Han [9] & 2005 & China & Asian & PB & $210 / 427$ & 0.037 & 6 \\
\hline Piller [43] & 2006 & Germany & Caucasian & PB & $608 / 1298$ & 0.062 & 7 \\
\hline Chakraborty [44] & 2007 & India & Asian & PB & $186 / 212$ & 0.550 & 6 \\
\hline Setiawan [45] & 2007 & USA & Mixed & PB & $5147 / 6882$ & 0.312 & 7 \\
\hline Chen [46] & 2008 & USA & Caucasian & PB & $1037 / 1096$ & 0.884 & 7 \\
\hline Sakoda [47] & 2008 & China & Asian & PB & $615 / 877$ & 0.232 & 7 \\
\hline Zhang [48] & 2008 & China & Asian & PB & $299 / 342$ & 0.454 & 7 \\
\hline Samson [49] & 2009 & India & Asian & PB & $250 / 500$ & 0.720 & 7 \\
\hline Sangrajrang [50] & 2009 & Thailand & Asian & HB & $564 / 489$ & 0.418 & 7 \\
\hline Sobczuk [51] & 2009 & Poland & Caucasian & PB & $100 / 106$ & 0.503 & 6 \\
\hline Antognelli [52] & 2009 & Italy & Caucasian & PB & $547 / 544$ & 0.982 & 7 \\
\hline Hosseini [53] & 2009 & Iran & Caucasian & $\mathrm{HB}$ & $53 / 53$ & 0.057 & 5 \\
\hline Jakubowska [54] & 2009 & Poland & Caucasian & HB & $319 / 290$ & 0.519 & 6 \\
\hline MARIE-GENICA [55] & 2009 & Germany & Caucasian & $\mathrm{PB}$ & $3145 / 5487$ & 0.254 & 7 \\
\hline Kato [56] & 2009 & USA & African & $\mathrm{PB}$ & $184 / 189$ & 0.152 & 6 \\
\hline Tuzuner [57] & 2010 & Turkey & Caucasian & PB & $55 / 91$ & 0.466 & 5 \\
\hline Syamala [58] & 2010 & India & Asian & HB & $359 / 367$ & 0.464 & 7 \\
\hline
\end{tabular}




\begin{tabular}{llcccccc} 
Surekha [59] & 2010 & India & Asian & PB & $249 / 249$ & 0.949 & 7 \\
Iwasaki [60] & 2010 & Japan & Asian & HB & $388 / 388$ & 0.299 & 6 \\
Iwasaki [60] & 2010 & Brazil & Asian & HB & $78 / 79$ & 0.144 & 6 \\
Iwasaki [60] & 2010 & Brazil & Caucasian & HB & $379 / 379$ & 0.039 & 6 \\
Kaufman [61] & 2011 & Mixed & Mixed & HB & $1175 / 829$ & 0.944 & 7 \\
Cribb [10] & 2011 & Canada & Caucasian & HB & $207 / 621$ & 0.033 & 6 \\
Ghisari [62] & 2014 & Inuit & Asian & PB & $30 / 113$ & 0.882 & 5 \\
Chattopadhyay [63] & 2014 & India & Asian & PB & $360 / 360$ & 0.692 & 7 \\
Karakus [64] & 2015 & Turkey & Caucasian & PB & $199 / 197$ & 0.934 & 6 \\
Farzaneh [65] & 2016 & Iranian & Caucasian & PB & $124 / 100$ & 0.189 & 6 \\
\hline
\end{tabular}

HWE: Hardy-Weinberg equilibrium for controls. PB: population-based study. HB: hospital-based study.

Table 2: Genotype distribution of the CYP17 (rs743572) polymorphism in cases and controls among overall populations

\begin{tabular}{|c|c|c|c|c|c|c|c|c|}
\hline \multirow{3}{*}{ First author } & \multicolumn{8}{|c|}{ Genotype (N) } \\
\hline & \multicolumn{4}{|c|}{ Case } & \multicolumn{4}{|c|}{ Control } \\
\hline & Total & $\mathrm{CC}$ & CT & TT & Total & $\mathrm{CC}$ & CT & TT \\
\hline Dunning & 835 & 130 & 402 & 303 & 591 & 85 & 277 & 229 \\
\hline Weston & 103 & 18 & 47 & 38 & 205 & 35 & 93 & 77 \\
\hline Weston & 20 & 3 & 10 & 7 & 35 & 2 & 18 & 15 \\
\hline Helzlsouer & 109 & 21 & 47 & 41 & 113 & 18 & 58 & 37 \\
\hline Bergman & 109 & 15 & 62 & 32 & 117 & 9 & 55 & 53 \\
\hline Haiman & 463 & 73 & 212 & 178 & 618 & 94 & 307 & 217 \\
\hline Huang & 123 & 44 & 54 & 25 & 126 & 35 & 63 & 28 \\
\hline Young & 39 & 5 & 13 & 21 & 58 & 7 & 28 & 23 \\
\hline Kristensen & 510 & 67 & 241 & 202 & 201 & 26 & 101 & 74 \\
\hline Hamajima & 144 & 20 & 83 & 41 & 166 & 27 & 95 & 44 \\
\hline Kuligina & 240 & 47 & 111 & 82 & 182 & 44 & 77 & 61 \\
\hline Mitrunen & 479 & 53 & 227 & 199 & 480 & 60 & 220 & 200 \\
\hline Feigelson & 850 & 149 & 409 & 292 & 1508 & 227 & 739 & 542 \\
\hline Gudmundsdottir & 500 & 60 & 247 & 193 & 395 & 66 & 173 & 156 \\
\hline $\mathrm{Wu}$ & 188 & 69 & 82 & 37 & 671 & 229 & 333 & 109 \\
\hline Ambrosone & 207 & 15 & 83 & 109 & 188 & 22 & 71 & 95 \\
\hline Tan & 250 & 89 & 115 & 46 & 250 & 89 & 110 & 51 \\
\hline Hefler & 388 & 75 & 186 & 127 & 1698 & 287 & 804 & 607 \\
\hline Ahsan & 313 & 49 & 155 & 109 & 271 & 51 & 140 & 80 \\
\hline Chacko & 140 & 6 & 40 & 94 & 140 & 3 & 22 & 115 \\
\hline Einarsdo'ttir & 1499 & 238 & 711 & 550 & 1338 & 212 & 638 & 488 \\
\hline Shin & 462 & 127 & 223 & 112 & 337 & 115 & 152 & 70 \\
\hline Verla-Tebit & 527 & 103 & 244 & 180 & 904 & 157 & 424 & 323 \\
\hline Hopper & 1404 & 230 & 621 & 553 & 788 & 113 & 364 & 311 \\
\hline Onland-More & 335 & 44 & 140 & 151 & 373 & 50 & 157 & 166 \\
\hline Han & 210 & 52 & 105 & 53 & 427 & 92 & 235 & 100 \\
\hline Piller & 608 & 119 & 289 & 200 & 1298 & 236 & 596 & 466 \\
\hline Chakraborty & 186 & 59 & 98 & 29 & 212 & 45 & 110 & 57 \\
\hline Setiawan & 5147 & 833 & 2445 & 1869 & 6882 & 1070 & 3338 & 2474 \\
\hline
\end{tabular}




\begin{tabular}{|c|c|c|c|c|c|c|c|c|}
\hline Chen & 1037 & 168 & 506 & 363 & 1096 & 175 & 523 & 398 \\
\hline Sakoda & 615 & 216 & 297 & 102 & 877 & 298 & 441 & 138 \\
\hline Zhang & 299 & 84 & 168 & 47 & 242 & 73 & 125 & 44 \\
\hline Samson & 250 & 32 & 91 & 127 & 500 & 54 & 226 & 220 \\
\hline Sangrajrang & 564 & 96 & 281 & 187 & 489 & 92 & 230 & 167 \\
\hline Sobczuk & 100 & 46 & 44 & 10 & 106 & 34 & 55 & 17 \\
\hline Antognelli & 547 & 60 & 258 & 229 & 544 & 68 & 249 & 227 \\
\hline Hosseini & 53 & 6 & 29 & 18 & 53 & 13 & 33 & 7 \\
\hline Jakubowska & 319 & 45 & 166 & 108 & 290 & 54 & 136 & 100 \\
\hline $\begin{array}{l}\text { MARIE- } \\
\text { GENICA }\end{array}$ & 3145 & 529 & 1573 & 1043 & 5487 & 941 & 2712 & 1834 \\
\hline Kato & 184 & 32 & 82 & 70 & 189 & 29 & 78 & 82 \\
\hline Tuzuner & 55 & 10 & 27 & 18 & 91 & 9 & 44 & 38 \\
\hline Syamala & 359 & 44 & 152 & 163 & 367 & 41 & 154 & 172 \\
\hline Surekha & 249 & 9 & 69 & 171 & 249 & 16 & 95 & 138 \\
\hline Iwasaki & 388 & 88 & 189 & 111 & 388 & 84 & 182 & 122 \\
\hline Iwasaki & 78 & 13 & 48 & 17 & 79 & 23 & 33 & 23 \\
\hline Iwasaki & 379 & 59 & 185 & 135 & 379 & 49 & 200 & 130 \\
\hline Kaufman & 1175 & 171 & 581 & 423 & 829 & 124 & 392 & 313 \\
\hline Cribb & 207 & 23 & 85 & 99 & 621 & 89 & 259 & 273 \\
\hline Ghisari & 30 & 6 & 12 & 12 & 113 & 32 & 57 & 24 \\
\hline Chattopadhyay & 360 & 14 & 116 & 230 & 360 & 7 & 93 & 260 \\
\hline Karakus & 199 & 18 & 79 & 102 & 197 & 15 & 78 & 104 \\
\hline Farzaneh & 124 & 22 & 70 & 32 & 100 & 17 & 56 & 27 \\
\hline
\end{tabular}

Table 3: Genotype distribution of the CYP17 (rs743572) polymorphism in cases and controls among premenopausal women and postmenopausal women

\begin{tabular}{|c|c|c|c|c|c|c|c|c|}
\hline \multirow{3}{*}{ First author } & \multicolumn{8}{|c|}{ Genotype $(N)$} \\
\hline & \multicolumn{4}{|c|}{ Case } & \multicolumn{4}{|c|}{ Control } \\
\hline & Total & $\mathrm{CC}$ & CT & TT & Total & $\mathrm{CC}$ & CT & TT \\
\hline Helzlsouer & 24 & 4 & 9 & 11 & 25 & 4 & 13 & 8 \\
\hline Bergman & 109 & 15 & 62 & 32 & 117 & 9 & 55 & 53 \\
\hline Mitrunen & 163 & 15 & 71 & 77 & 203 & 27 & 88 & 88 \\
\hline $\mathrm{Wu}$ & 57 & 24 & 20 & 13 & 203 & 66 & 100 & 37 \\
\hline Ambrosone & 96 & 7 & 31 & 58 & 86 & 10 & 28 & 48 \\
\hline Verla-Tebit & 527 & 103 & 244 & 180 & 904 & 157 & 424 & 323 \\
\hline Chen & 334 & 55 & 153 & 126 & 373 & 69 & 174 & 130 \\
\hline Samson & 115 & 16 & 40 & 59 & 303 & 31 & 145 & 127 \\
\hline Antognelli & 187 & 18 & 81 & 88 & 230 & 31 & 99 & 100 \\
\hline Kato & 75 & 12 & 27 & 36 & 74 & 13 & 30 & 31 \\
\hline Zhang & 150 & 38 & 87 & 25 & 124 & 37 & 67 & 20 \\
\hline Tan & 95 & 32 & 45 & 18 & 97 & 30 & 40 & 27 \\
\hline Han & 117 & 25 & 61 & 31 & 163 & 36 & 85 & 42 \\
\hline Helzlsouer & 85 & 17 & 38 & 30 & 88 & 14 & 45 & 29 \\
\hline Mitrunen & 316 & 38 & 156 & 122 & 277 & 33 & 132 & 112 \\
\hline
\end{tabular}




\begin{tabular}{|c|c|c|c|c|c|c|c|c|}
\hline $\mathrm{Wu}$ & 131 & 45 & 62 & 24 & 468 & 163 & 233 & 72 \\
\hline Ambrosone & 111 & 8 & 52 & 51 & 102 & 12 & 43 & 47 \\
\hline Einarsdo'ttir & 1499 & 238 & 711 & 550 & 1338 & 212 & 638 & 488 \\
\hline $\begin{array}{l}\text { Onland- } \\
\text { More }\end{array}$ & 335 & 44 & 140 & 151 & 373 & 50 & 157 & 166 \\
\hline Chen & 680 & 111 & 339 & 230 & 677 & 96 & 333 & 248 \\
\hline Samson & 134 & 16 & 50 & 68 & 197 & 23 & 99 & 75 \\
\hline Antognelli & 360 & 42 & 177 & 141 & 314 & 37 & 150 & 127 \\
\hline Kato & 109 & 20 & 55 & 34 & 115 & 16 & 48 & 51 \\
\hline Zhang & 146 & 44 & 80 & 22 & 118 & 36 & 58 & 24 \\
\hline Tan & 155 & 57 & 70 & 28 & 153 & 59 & 70 & 24 \\
\hline Han & 93 & 27 & 44 & 22 & 264 & 56 & 150 & 58 \\
\hline
\end{tabular}

Table 4: Meta-analysis results among overall populations

\begin{tabular}{|c|c|c|c|c|c|c|c|c|}
\hline \multirow{2}{*}{ Comparisons } & \multirow{2}{*}{ OR } & \multirow{2}{*}{ 95\% CI } & \multirow{2}{*}{$P(\mathrm{OR})$} & \multicolumn{2}{|c|}{ Heterogeneity } & \multirow{2}{*}{$\begin{array}{c}\text { Effects } \\
\text { model }\end{array}$} & \multirow{2}{*}{$P$ (Begg) } & \multirow{2}{*}{$P$ (Egger) } \\
\hline & & & & $\bar{P}$ & $P$ & & & \\
\hline \multicolumn{9}{|l|}{ Total } \\
\hline T VS C & 0.99 & $0.96-1.01$ & 0.281 & $37.1 \%$ & 0.005 & $\mathrm{R}$ & 0.856 & 0.766 \\
\hline TT VS CC & 0.99 & $0.98-1.01$ & 0.309 & $18.6 \%$ & 0.127 & $\mathrm{~F}$ & 0.987 & 0.408 \\
\hline TC VS CC & 0.98 & $0.93-1.03$ & 0.365 & $0.80 \%$ & 0.457 & $\mathrm{~F}$ & 0.825 & 0.563 \\
\hline $\mathrm{TT}+\mathrm{TC}$ VS CC & 0.98 & $0.93-1.02$ & 0.287 & $15.0 \%$ & 0.182 & $\mathrm{~F}$ & 0.975 & 0.574 \\
\hline TT VS TC+CC & 0.99 & $0.95-1.02$ & 0.463 & $30.5 \%$ & 0.022 & $\mathrm{R}$ & 1.000 & 0.902 \\
\hline
\end{tabular}

Stratification by ethnicity

\section{Caucasian}

\begin{tabular}{lcccccccc} 
T VS C & 0.99 & $0.96-1.03$ & 0.673 & $11.5 \%$ & 0.294 & $\mathrm{~F}$ & - & - \\
TT VS CC & 0.99 & $0.93-1.06$ & 0.804 & $10.4 \%$ & 0.233 & $\mathrm{~F}$ & - & - \\
TC VS CC & 1.00 & $0.94-1.07$ & 0.936 & $0.00 \%$ & 0.467 & $\mathrm{~F}$ & - & - \\
TT+TC VS CC & 1.00 & $0.94-1.06$ & 0.604 & $11.1 \%$ & 0.301 & $\mathrm{~F}$ & - & - \\
TT VS TC+CC & 0.99 & $0.94-1.04$ & 0.907 & $0.00 \%$ & 0.596 & $\mathrm{~F}$ & - & - \\
Asian & & & & & & & & - \\
T VS C & 0.97 & $0.89-1.06$ & 0.574 & $60.8 \%$ & 0.023 & $\mathrm{R}$ & - & - \\
TT VS CC & 0.99 & $0.95-1.02$ & 0.483 & $33.9 \%$ & 0.075 & $\mathrm{R}$ & - & - \\
TC VS CC & 0.97 & $0.88-1.07$ & 0.525 & $11.4 \%$ & 0.282 & $\mathrm{~F}$ & - & - \\
TT+TC VS CC & 0.97 & $0.88-1.06$ & 0.479 & $29.1 \%$ & 0.114 & $\mathrm{~F}$ & - & - \\
TT VS TC+CC & 0.97 & $0.84-1.11$ & 0.652 & $60.0 \%$ & 0.000 & $\mathrm{R}$ & - & - \\
African & & & & & & & & - \\
T VS C & 0.83 & $0.63-1.10$ & 0.198 & $0.0 \%$ & 0.621 & $\mathrm{~F}$ & - & - \\
TT VS CC & 0.72 & $0.41-1.27$ & 0.255 & $0.0 \%$ & 0.393 & $\mathrm{~F}$ & - & - \\
TC VS CC & 0.88 & $0.50-1.54$ & 0.654 & $0.0 \%$ & 0.363 & $\mathrm{~F}$ & - & - \\
TT+TC VS CC & 0.80 & $0.47-1.36$ & 0.408 & $0.0 \%$ & 0.358 & $\mathrm{~F}$ & - & - \\
TT VS TC+CC & 0.79 & $0.54-1.17$ & 0.237 & $0.0 \%$ & 0.859 & $\mathrm{~F}$ & - & - \\
Stratification by Source & & & & & & & & \\
PB & & & & & & & & \\
T VS C & 0.98 & $0.95-1.00$ & 0.102 & $35.2 \%$ & 0.021 & $\mathrm{R}$ & - & - \\
TT VS CC & 0.95 & $0.90-1.01$ & 0.073 & $18.7 \%$ & 0.165 & $\mathrm{~F}$ & - & - \\
& & & & & & & - \\
\hline
\end{tabular}




\begin{tabular}{|c|c|c|c|c|c|c|c|c|}
\hline TC VS CC & 0.95 & $0.90-1.00$ & 0.047 & $0.0 \%$ & 0.726 & $\mathrm{~F}$ & - & - \\
\hline $\mathrm{TT}+\mathrm{TC}$ VS CC & 0.95 & $0.91-1.00$ & 0.034 & $1.8 \%$ & 0.439 & $\mathrm{~F}$ & - & - \\
\hline TT VS TC+CC & 0.99 & $0.95-1.02$ & 0.474 & $32.4 \%$ & 0.033 & $\mathrm{~F}$ & - & - \\
\hline \multicolumn{9}{|l|}{ HB } \\
\hline T VS C & 1.03 & $0.97-1.09$ & 0.299 & $38.9 \%$ & 0.057 & $\mathrm{R}$ & - & - \\
\hline TT VS CC & 1.10 & $0.97-1.24$ & 0.128 & $18.2 \%$ & 0.245 & $\mathrm{~F}$ & - & - \\
\hline TC VS CC & 1.14 & $1.02-1.28$ & 0.024 & $0.0 \%$ & 0.548 & $\mathrm{~F}$ & - & - \\
\hline $\mathrm{TT}+\mathrm{TC}$ VS CC & 1.13 & $1.01-1.26$ & 0.031 & $8.6 \%$ & 0.355 & $\mathrm{~F}$ & - & - \\
\hline TT VS TC+CC & 0.99 & $0.91-1.08$ & 0.847 & $30.6 \%$ & 0.118 & $\mathrm{~F}$ & - & - \\
\hline \multicolumn{9}{|c|}{ Stratification by HWE } \\
\hline \multicolumn{9}{|l|}{ Yes } \\
\hline T VS C & 0.99 & $0.96-1.01$ & 0.250 & $41.2 \%$ & 0.002 & $\mathrm{R}$ & - & - \\
\hline TT VS CC & 0.97 & $0.93-1.02$ & 0.288 & $26.6 \%$ & 0.050 & $\mathrm{R}$ & - & - \\
\hline TC VS CC & 0.99 & $0.98-1.01$ & 0.421 & $0.0 \%$ & 0.501 & $\mathrm{~F}$ & - & - \\
\hline $\mathrm{TT}+\mathrm{TC}$ VS CC & 0.99 & $0.99-1.02$ & 0.264 & $15.6 \%$ & 0.180 & $\mathrm{~F}$ & - & - \\
\hline TT VS TC+CC & 0.98 & $0.95-1.02$ & 0.381 & $35.3 \%$ & 0.010 & $\mathrm{R}$ & - & - \\
\hline
\end{tabular}

F: fixed effects model; R: random effects model.

Table 5: Meta-analysis results among premenopausal women and postmenopausal women

\begin{tabular}{|c|c|c|c|c|c|c|c|c|}
\hline \multirow{2}{*}{ Comparisons } & \multirow{2}{*}{ OR } & \multirow{2}{*}{$95 \%$ CI } & \multirow{2}{*}{$P(\mathrm{OR})$} & \multicolumn{2}{|c|}{ Heterogeneity } & \multirow{2}{*}{$\begin{array}{c}\text { Effects } \\
\text { model }\end{array}$} & \multirow{2}{*}{$P$ (Begg) } & \multirow{2}{*}{$P$ (Egger) } \\
\hline & & & & $r^{2}$ & $P$ & & & \\
\hline \multicolumn{9}{|l|}{ Premenopausal } \\
\hline T VS C & 1.02 & $0.93-1.10$ & 0.717 & $17.6 \%$ & 0.267 & $\mathrm{~F}$ & - & - \\
\hline TT VS CC & 1.01 & $0.85-1.20$ & 0.885 & $6.4 \%$ & 0.383 & $\mathrm{~F}$ & - & - \\
\hline TC VS CC & 0.97 & $0.83-1.14$ & 0.709 & $0.0 \%$ & 0.492 & $\mathrm{~F}$ & - & - \\
\hline $\mathrm{TT}+\mathrm{TC}$ VS CC & 1.04 & $0.92-1.18$ & 0.513 & $21.4 \%$ & 0.227 & $\mathrm{~F}$ & - & - \\
\hline TT VS TC+CC & 0.95 & $0.81-1.10$ & 0.467 & $29.3 \%$ & 0.151 & $\mathrm{~F}$ & - & - \\
\hline \multicolumn{9}{|c|}{ Postmenopausal } \\
\hline T VS C & 1.10 & $1.03-1.17$ & 0.003 & $10.6 \%$ & 0.339 & $\mathrm{~F}$ & - & - \\
\hline TT VS CC & 0.96 & $0.84-1.10$ & 0.539 & $0.0 \%$ & 0.835 & $\mathrm{~F}$ & - & - \\
\hline TC VS CC & 0.96 & $0.85-1.08$ & 0.478 & $0.0 \%$ & 0.902 & $\mathrm{~F}$ & - & - \\
\hline $\mathrm{TT}+\mathrm{TC}$ VS CC & 0.96 & $0.85-1.08$ & 0.467 & $0.0 \%$ & 0.930 & $\mathrm{~F}$ & - & - \\
\hline TT VS TC $+\mathrm{CC}$ & 0.99 & $0.90-1.08$ & 0.796 & $8.9 \%$ & 0.357 & $\mathrm{~F}$ & - & - \\
\hline
\end{tabular}

F: fixed effects model; R: random effects model.

between rs743572 and $\mathrm{BC}$ risk we performed this metaanalysis whose included research studies identified in the PubMed, EMBASE and the Cochrane.

In overall populations, our results indicate no significant correlation between $\mathrm{rs} 743572$ and the risk of BC. Similar results could be obtained when stratified by ethnicity in all ethnic groups. In addition, confining the analysis to the researches with control groups in consistent with HWE, we also observed no correlation between rs743572 and risk of BC. Nevertheless, meaningful correlation was showed between rs743572 and breast neoplasm risk in Russian individuals [10]. There were three meta-analyses, all published in 2010, including 24-43 papers from different populations and demonstrated no association between the rs 743572 and $\mathrm{BC}$, which further demonstrate that our results are credible $[12,13,14]$.

Estrogen is mainly produced in the ovaries and mammary glands among premenopausal women. However, in postmenopausal individuals, adipose tissue mainly acts as an important part in estrogen biosynthesis $[15,16]$. Several studies have reported conflicting results of menopausal and CYP17 polymorphism: the study 

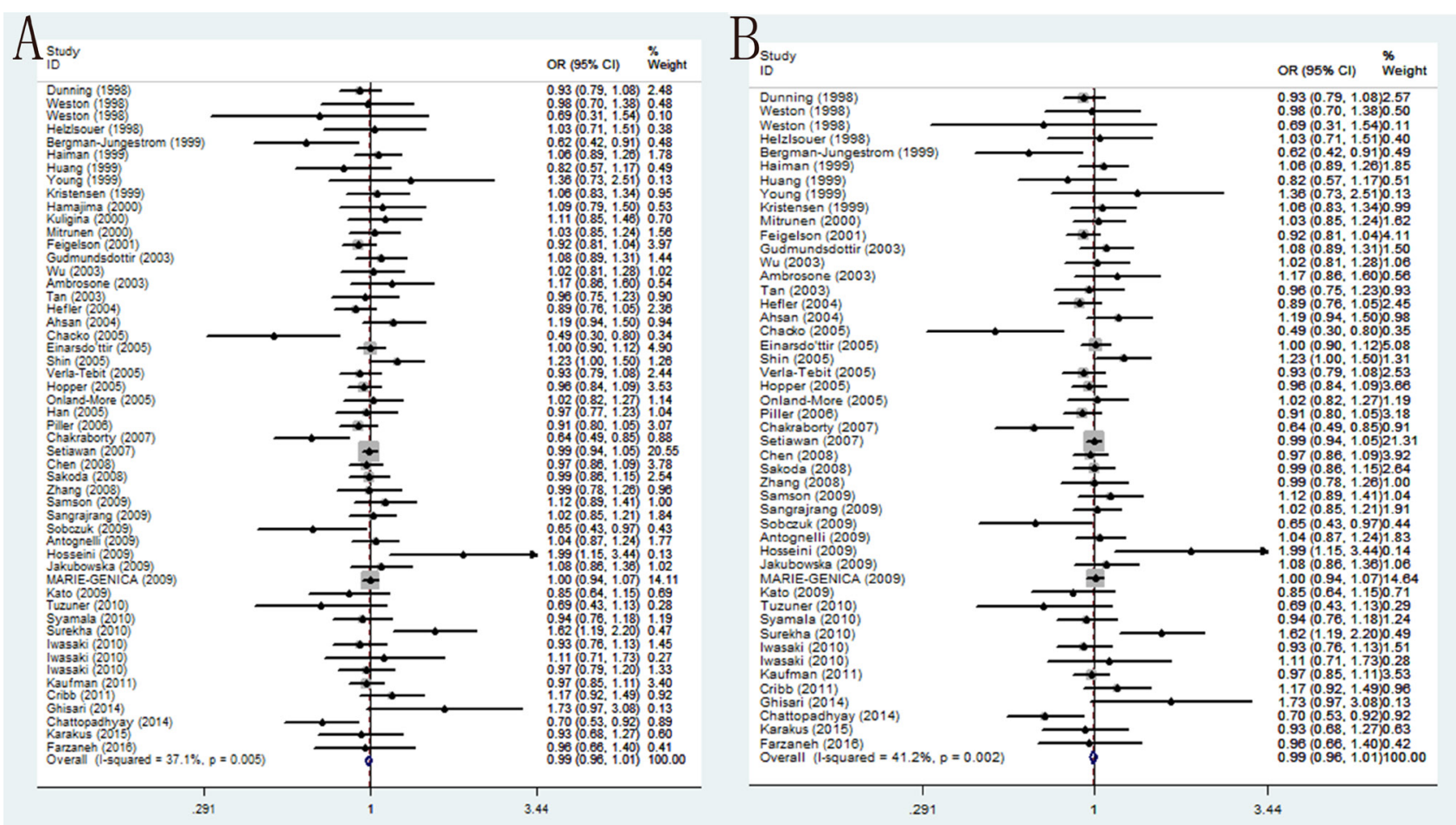

Figure 2: Forest plots of associations between rs743572 and breast cancer risk. (A) the overall populations in the allele contrast genetic model; (B) limiting the analysis to studies with controls in agreement with HWE under the allele contrast genetic model.

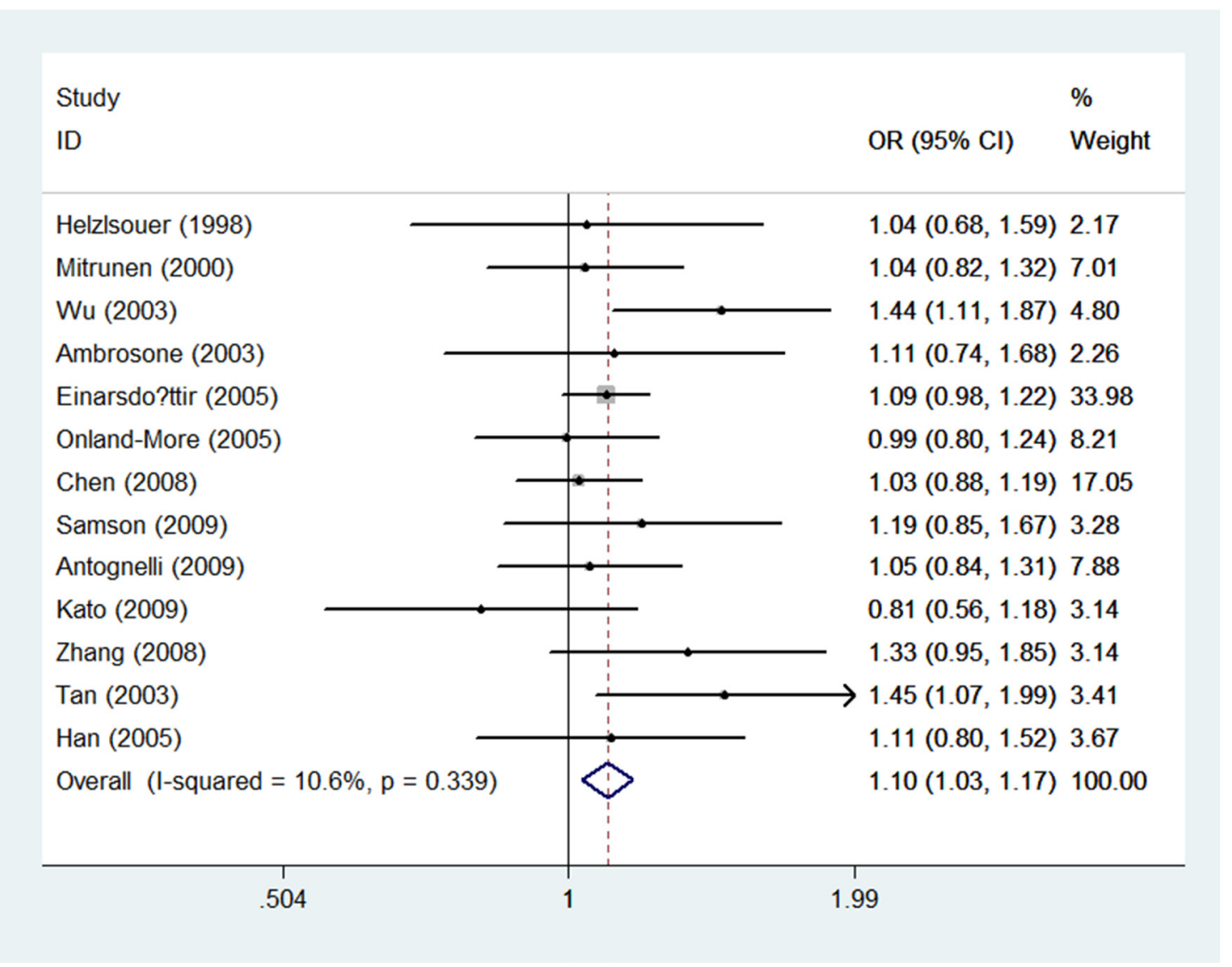

Figure 3: Forest plots of associations between rs743572 and breast cancer risk among postmenopausal women in the allele contrast genetic model. 
by Dunning et al. [17] showed the association between increased A2 genotype and premenopausal breast cancer; while Feigelson et al. [18] reported increasing frequency of A2 genotype associated with postmenopausal BC patients. We observed that rs 743572 was correlated with an increasing $\mathrm{BC}$ risk among postmenopausal women under the allele contrast genetic model, but not in other models; however, no association was found in premenopausal women. Previous published meta- analysis reported that no association existed both in postmenopausal women and among premenopausal women [12, 13, 14]. Compared with them, our study used five genetic models to reduce the probability of class I errors, so our result was more reliable.

Unavoidable, there are some limitations in metaanalysis. First, breast cancer is a multifactorial disease involving genetic and environmental interactions; however, it was still not addressed the impact of geneenvironmental interactions in this meta-analysis [19]. Second, the detailed individual information in some studies was unknown; thus, we could not assess the susceptibility of breast cancer according to other risk factors including obesity, family history, radiation therapy in young age, history of pregnancy, breast-feeding, hormone therapy and so on [20]. Last, there are only two studies about Africans, more well designed studies with different population should be performed to make more persuasive conclusions. In summary, our results indicate that rs 743572 could increase risk of BC in postmenopausal individuals, but not in premenopausal women and the general population. Further multicenter research with complete risk factors are required to validate the potential role of rs 743572 polymorphism in BC. More multicenter studies and complete risk factors are needed to further confirm the possible role of rs743572 polymorphism in the occurrence and development of breast cancer.

\section{MATERIALS AND METHODS}

\section{Literature and search strategy}

We searched the PubMed, EMBASE and Cochrane databases for studies performed prior to March 7, 2017 that reported an association between rs743572 SNP and breast cancer risk. There were no language restrictions in our searching process. The searching strategy was as follow: (breast cancer OR breast carcinoma) AND (polymorphism OR variant OR genotype OR SNP) AND (CYP17 OR CYP17A1 OR P450c17). Besides, the references of the retrieved studies were also reviewed to identify additional eligible studies.

\section{Inclusion criteria}

The included studies must meet the following criteria: (1) case-control design; (2) investigating the association between CYP17 T-34C polymorphism and breast cancer risk; (3) sufficient genotyping data that could be used to calculate odds ratios (ORs) and 95\% confidence intervals (CIs); (4) all the breast cancer subjects in case groups must be pathologically confirmed. The exclusion criteria were: (1) not case-control studies; (2) review article or commentary; (3) duplicate studies; (4) studies lacking relevant data.

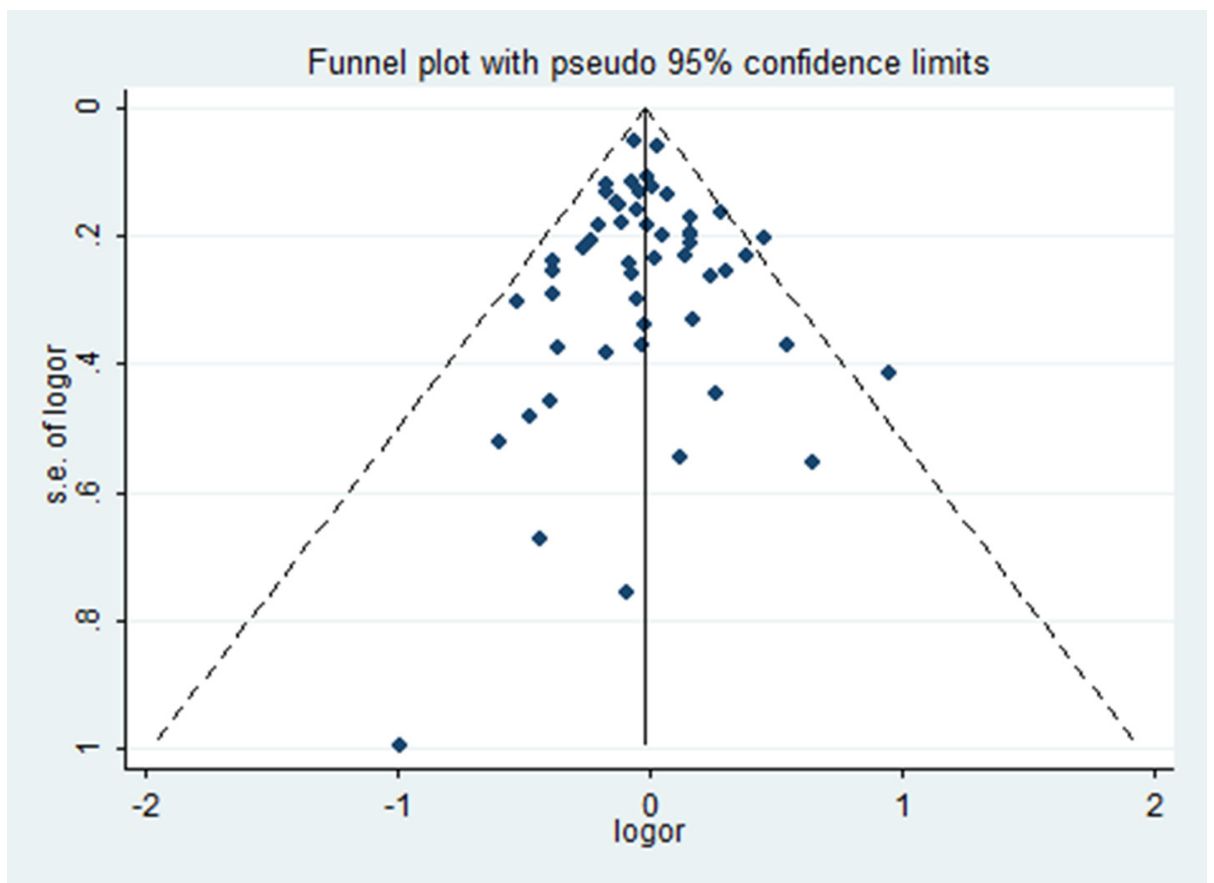

Figure 4: Funnel plots of rs743572 and breast cancer risk in the heterozygote genetic model. 


\section{Data extraction}

Two reviewers independently extracted the relevant data from the included studies, and discrepancies were resolved during a discussion with a third author. The following information was extracted: the first author, year of publication, country, ethnicity, source of controls, number of cases and controls, and $P$ value for Hardy-Weinberg equilibrium (HWE). In addition, we also evaluated the methodological quality of included studies based on Newcastle-Ottawa Scale (NOS), which scored studies according to three aspects: selection, comparability, and exposure. Therefore, all studies could be divided into three categories: "low quality" studies (score 0-3); "moderate quality" studies (score 4-6); "high quality" studies (score 7-9).

\section{Statistical analysis}

The association between CYP17 T-34C polymorphism and $\mathrm{BC}$ susceptibility was measured by pooled odds ratios (ORs) and 95\% confidence intervals (CIs) in five genetic models, including an allele contrast genetic model, a homozygote genetic model, a heterozygote genetic model, a dominant genetic model, and a recessive genetic model. Pooled ORs were performed for homozygote comparison (TT vs. CC for rs743572), heterozygote comparison (TC vs. CC for rs743572), dominant model (TT/TC vs. CC for rs743572), recessive model (TT vs. TC/CC for rs743572) and allelic model ( $\mathrm{T}$ vs. C for rs 743572) respectively. Statistical heterogeneity was evaluated by $I^{2}$ test and $Q$ test, $P<0.05$ was considered statistically significant. For ${ }^{2}$ test, the criteria for heterogeneity were as follows: $P<25 \%$, no heterogeneity; $25 \%-75 \%$, moderate heterogeneity; $P^{2}>75 \%$, high heterogeneity. If the $P$ value of $Q$ test was $<0.1$, the random-effects model was used; otherwise, the fixed-effects model was applied. Sensitivity analysis was performed by excluding one study at a time to assess the influence of each study on the pooled ORs. Begg's funnel plot and Egger's tests were used to examine publication bias and to evaluate the stability of the results by sensitivity analysis. The $P$ value for Hardy-Weinberg equilibrium (HWE) in controls of every included study was calculated by Chi-square test. Subgroup analysis was performed according to ethnicity. All statistical analyses were performed using STATA version 10.0 software (StataCorp LP, College Station, TX, USA). All $P$ values were two sided, and $P<0.05$ was considered statistically significant.

\section{CONFLICTS OF INTEREST}

The authors declare that no conflicts of interest exist.

\section{REFERENCES}

1. Ghoncheh M, Mirzaei M, Salehiniya H. Incidence and Mortality of Breast Cancer and their Relationship with the
Human Development Index (HDI) in the World in 2012. Asian Pac J Cancer Prev. 2015; 16:8439-8443.

2. Eccles DM, Pichert G. Familial non-BRCA1/BRCA2associated breast cancer. Lancet Oncol. 2005; 6:705-711.

3. LaMarca HL, Rosen JM. Estrogen regulation of mammary gland development and breast cancer: amphiregulin takes center stage. Breast cancer Res. 2007; 9:304.

4. Mitrunen K, Jourenkova N, Kataja V, Eskelinen M, Kosma VM, Benhamou S, Vainio H, Uusitupa M, Hirvonen A. Steroid metabolism gene CYP17 polymorphism and the development of breast cancer. Cancer Epidem Biomar Prev. 2000; 9:1343-1348.

5. Khatri Y, Gregory MC, Grinkova YV, Denisov IG, Sligar SG. Active site proton delivery and the lyase activity of human CYP17A1. Biochem Bioph Res Commun. 2014; 443:179-184.

6. Baston E, Leroux FR. Inhibitors of steroidal cytochrome p450 enzymes as targets for drug development. Recent Pat Anti-Canc. 2007; 2:31-58.

7. Tuzuner BM, Ozturk T, Kisakesen HI, Ilvan S, Zerrin C, Ozturk O, Isbir T. CYP17 (T-34C) and CYP19 (Trp39Arg) polymorphisms and their cooperative effects on breast cancer susceptibility. In vivo (Athens, Greece). 2010; 24:71-74.

8. Feigelson HS, McKean-Cowdin R, Pike MC, Coetzee GA, Kolonel LN, Nomura AM, Le Marchand L, Henderson BE. Cytochrome P450c17alpha gene (CYP17) polymorphism predicts use of hormone replacement therapy. Cancer Res. 1999; 59:3908-3910.

9. Han DF, Zhou X, Hu MB, Xie W, Mao ZF, Chen DE, Liu F, Zheng F. Polymorphisms of estrogen-metabolizing genes and breast cancer risk: a multigenic study. Chinese Med J. 2005; 118:1507-1516.

10. Cribb AE, Joy Knight M, Guernsey J, Dryer D, Hender K, Shawwa A, Tesch M, Saleh TM. CYP17, catechol-omethyltransferase, and glutathione transferase M1 genetic polymorphisms, lifestyle factors, and breast cancer risk in women on Prince Edward Island. Breast J. 2011; 17:24-31.

11. Chen WY. Exogenous and endogenous hormones and breast cancer. Best Pract Res Clin Endocrinol Metab. 2008; 22:573-585.

12. Yao L, Fang F, Wu Q, Yang Z, Zhong Y, Yu L. No association between CYP17 T-34C polymorphism and breast cancer risk: a meta-analysis involving 58,814 subjects. Breast Cancer Res Treat. 2010; 122:221-227.

13. Mao C, Wang XW, He BF, Qiu LX, Liao RY, Luo RC, Chen Q. Lack of association between CYP17 MspA1 polymorphism and breast cancer risk: a meta-analysis of 22,090 cases and 28,498 controls. Breast Cancer RS Treat. 2010; 122:259-265.

14. Chen Y, Pei J. Factors influencing the association between CYP17 T34C polymorphism and the risk of breast cancer: meta-regression and subgroup analysis. Breast cancer RS Treat. 2010; 122:471-481. 
15. Gago-Dominguez M, Castelao JE, Pike MC, Sevanian A, Haile RW. Role of lipid peroxidation in the epidemiology and prevention of breast cancer. Cancer Epidem Biomar Prev. 2005; 14:2829-2839.

16. Miyoshi Y, Funahashi T, Kihara S, Taguchi T, Tamaki Y, Matsuzawa Y, Noguchi S. Association of serum adiponectin levels with breast cancer risk. Clin Cancer Res. 2003; 9:5699-5704.

17. Dunning AM, Healey CS, Pharoah PD, Foster NA, Lipscombe JM, Redman KL, Easton DF, Day NE, Ponder BA. No association between a polymorphism in the steroid metabolism gene CYPI 7 and risk of breast cancer. Brit J Cancer. 1998; 77:2045-2047.

18. Feigelson HS, McKean-Cowdin R, Coetzee GA, Stram DO, Kolonel LN, Henderson BE. Building a multigenic model of breast cancer susceptibility: CYP17 and HSD17B1 are two important candidates. Cancer Res. 2001; 61:785-789.

19. Wu K, Su D, Lin K, Luo J, Au WW. XRCC1 Arg399Gln gene polymorphism and breast cancer risk: a meta-analysis based on case-control studies. Asian Pac J Cancer Prev. 2011; 12:2237-2243.

20. Hill DA, Gilbert E, Dores GM, Gospodarowicz M, van Leeuwen FE, Holowaty E, Glimelius B, Andersson M, Wiklund T, Lynch CF, Van't Veer M, Storm H, Pukkala E, et al. Breast cancer risk following radiotherapy for Hodgkin lymphoma: modification by other risk factors. Blood. 2005; 106:3358-3365.

21. Weston A, Pan CF, Bleiweiss IJ, Ksieski HB, Roy N, Maloney N, Wolff MS. CYP17 genotype and breast cancer risk. Cancer Epidem Biomar Prev. 1998; 7:941-944.

22. Helzlsouer KJ, Huang HY, Strickland PT, Hoffman S, Alberg AJ, Comstock GW, Bell DA. Association between CYP17 polymorphisms and the development of breast cancer. Cancer Epidem Biomar Prev. 1998; 7:945-949.

23. Bergman-Jungestrom M, Gentile M, Lundin AC, Wingren S. Association between CYP17 gene polymorphism and risk of breast cancer in young women. Int J Cancer. 1999; 84:350-353.

24. Haiman CA, Hankinson SE, Spiegelman D, Colditz GA, Willett WC, Speizer FE, Kelsey KT, Hunter DJ. The relationship between a polymorphism in CYP17 with plasma hormone levels and breast cancer. Cancer Res. 1999; 59:1015-1020.

25. Huang CS, Chern HD, Chang KJ, Cheng CW, Hsu SM, Shen CY. Breast cancer risk associated with genotype polymorphism of the estrogen-metabolizing genes CYP17, CYP1A1, and COMT: a multigenic study on cancer susceptibility. Cancer Res. 1999; 59:4870-4875.

26. Young IE, Kurian KM, Annink C, Kunkler IH, Anderson VA, Cohen BB, Hooper ML, Wyllie AH, Steel CM. A polymorphism in the CYP17 gene is associated with male breast cancer. Brit J Cancer. 1999; 81:141-143.

27. Nedelcheva Kristensen V, Haraldsen EK, Anderson KB, Lonning PE, Erikstein B, Karesen R, Gabrielsen OS,
Borresen-Dale AL. CYP17 and breast cancer risk: the polymorphism in the $5^{\prime}$ flanking area of the gene does not influence binding to Sp-1. Cancer Res. 1999; 59:2825-2828.

28. Hamajima N, Iwata H, Obata Y, Matsuo K, Mizutani M, Iwase T, Miura S, Okuma K, Ohashi K, Tajima K. No association of the $5^{\prime}$ promoter region polymorphism of CYP17 with breast cancer risk in Japan. Jpn J Cancer Res. 2000; 91:880-885.

29. Kuligina ES, Togo AV, Suspitsin EN, Grigoriev MY, Pozharisskiy KM, Chagunava OL, Berstein LM, Theillet C, Hanson KP, Imyanitov EN. CYP17 polymorphism in the groups of distinct breast cancer susceptibility: comparison of patients with the bilateral disease vs. monolateral breast cancer patients vs. middle-aged female controls vs. elderly tumor-free women. Cancer Lett. 2000; 156:45-50.

30. Mitrunen K, Jourenkova N, Kataja V, Eskelinen M, Kosma VM, Benhamou S, Vainio H, Uusitupa M, Hirvonen A. Steroid metabolism gene CYP17 polymorphism and the development of breast cancer. Cancer Epidem Biomar Prev. 2000; 9:1343-1348.

31. Gudmundsdottir K, Thorlacius S, Jonasson JG, Sigfusson BF, Tryggvadottir L, Eyfjord JE. CYP17 promoter polymorphism and breast cancer risk in males and females in relation to BRCA2 status. Brit J Cancer. 2003; 88:933-936.

32. Wu AH, Seow A, Arakawa K, Van Den Berg D, Lee HP, Yu MC. HSD17B1 and CYP17 polymorphisms and breast cancer risk among Chinese women in Singapore. Int J Cancer. 2003; 104:450-457.

33. Ambrosone CB, Moysich KB, Furberg H, Freudenheim JL, Bowman ED, Ahmed S, Graham S, Vena JE, Shields PG. CYP17 genetic polymorphism, breast cancer, and breast cancer risk factors. Breast Cancer Res. 2003; 5:1-7.

34. Tan W, Qi J, Xing DY, Miao XP, Pan KF, Zhang L, Lin DX. [Relation between single nucleotide polymorphism in estrogen-m etabolizing genes COMT, CYP17 and breast cancer risk among Chinese women]. [Article in Chinese]. Chinese J Oncol. 2003; 25:453-456.

35. Hefler LA, Tempfer CB, Grimm C, Lebrecht A, Ulbrich E, Heinze G, Leodolter S, Schneeberger C, Mueller MW, Muendlein A, Koelbl H. Estrogen-metabolizing gene polymorphisms in the assessment of breast carcinoma risk and fibroadenoma risk in Caucasian women. Cancer. 2004; 101:264-269.

36. Ahsan H, Whittemore AS, Chen Y, Senie RT, Hamilton SP, Wang Q, Gurvich I, Santella RM. Variants in estrogenbiosynthesis genes CYP17 and CYP19 and breast cancer risk: a family-based genetic association study. Breast Cancer Res. 2004; 7:R71-81.

37. Chacko P, Rajan B, Mathew BS, Joseph T, Pillai MR. CYP17 and SULT1A1 Gene Polymorphisms in Indian Breast Cancer. Breast Cancer. 2004, 11:380-388.

38. Einarsdottir K, Rylander-Rudqvist T, Humphreys K, Ahlberg S, Jonasdottir G, Weiderpass E, Chia KS, Ingelman-Sundberg M, Persson I, Liu J, Hall P, Wedren S. 
CYP17 gene polymorphism in relation to breast cancer risk: a case-control study. Breast cancer Res. 2005; 7:R890-896.

39. Shin MH, Lee KM, Yang JH, Nam SJ, Kim JW, Yoo KY, Park SK, Noh DY, Ahn SH, Kim B, Kang D. Genetic polymorphism of CYP17 and breast cancer risk in Korean women. Exp Mol Med. 2005; 37:11-17.

40. Verla-Tebit E, Wang-Gohrke S, Chang-Claude J. CYP17 5'-UTR MspA1 polymorphism and the risk of premenopausal breast cancer in a German population-based case-control study. Breast Cancer Res. 2005; 7:R455-464.

41. Hopper JL, Hayes VM, Spurdle AB, Chenevix-Trench G, Jenkins MA, Milne RL, Dite GS, Tesoriero AA, McCredie MR, Giles GG, Southey MC. A protein-truncating mutation in CYP17A1 in three sisters with early-onset breast cancer. Hum Mutat. 2005; 26:298-302.

42. Onland-Moret NC, van Gils CH, Roest M, Grobbee DE, Peeters PH. Cyp17, urinary sex steroid levels and breast cancer risk in postmenopausal women. Cancer Epidem Biomar Prev. 2005; 14:815-820.

43. Piller R, Verla-Tebit E, Wang-Gohrke S, Linseisen J, Chang-Claude J. CYP17 genotype modifies the association between lignan supply and premenopausal breast cancer risk in humans. J Nutr. 2006; 136:1596-1603.

44. Chakraborty A, Murthy NS, Chintamani C, Bhatnagar D, Mohil RS, Sharma PC, Saxena S. CYP17 gene polymorphism and its association with high-risk north Indian breast cancer patients. J Hum Genet. 2007; 52:159-165.

45. Setiawan VW, Schumacher FR, Haiman CA, Stram DO, Albanes D, Altshuler D, Berglund G, Buring J, Calle EE, Clavel-Chapelon F, Cox DG, Gaziano JM, Hankinson SE, et al. CYP17 genetic variation and risk of breast and prostate cancer from the National Cancer Institute Breast and Prostate Cancer Cohort Consortium (BPC3). Cancer Epidem Biomar Prev. 2007; 16:2237-2246.

46. Chen Y, Gammon MD, Teitelbaum SL, Britton JA, Terry MB, Shantakumar S, Eng SM, Wang Q, Gurvich I, Neugut AI, Santella RM, Ahsan H. Estrogen-biosynthesis gene CYP17 and its interactions with reproductive, hormonal and lifestyle factors in breast cancer risk: results from the Long Island Breast Cancer Study Project. Carcinogenesis. 2008; 29:766-771.

47. Sakoda LC, Blackston C, Doherty JA, Ray RM, Lin MG, Stalsberg H, Gao DL, Feng Z, Thomas DB, Chen C. Polymorphisms in steroid hormone biosynthesis genes and risk of breast cancer and fibrocystic breast conditions in Chinese women. Cancer Epidem Biomar Prev. 2008; 17:1066-1073.

48. Zhang L, Gu L, Qian B, Hao X, Zhang W, Wei Q, Chen K. Association of genetic polymorphisms of ER-alpha and the estradiol-synthesizing enzyme genes CYP17 and CYP19 with breast cancer risk in Chinese women. Breast Cancer Res Treat. 2008; 114:327-338.

49. Samson M, Rama R, Swaminathan R, Sridevi V, Nancy KN, Rajkumar T. CYP17 (T34C), CYP19 (Trp39Arg), and
FGFR2 (C906T) polymorphisms and the risk of breast cancer in south Indian women. Asian Pac J Cancer Prev. 2009; 10:111-114.

50. Sangrajrang S, Sato Y, Sakamoto H, Ohnami S, Laird NM, Khuhaprema T, Brennan P, Boffetta P, Yoshida T. Genetic polymorphisms of estrogen metabolizing enzyme and breast cancer risk in Thai women. Int J Cancer. 2009; 125:837-843.

51. Sobczuk A, Romanowicz H, Fiks T, Polac I, Smolarz B. The CYP17 and CYP19 gene single nucleotide polymorphism in women with sporadic breast cancer. Polish J Pathol J. 2009; 60:163-167.

52. Antognelli C, Del Buono C, Ludovini V, Gori S, Talesa VN, Crino L, Barberini F, Rulli A. CYP17, GSTP1, PON1 and GLO1 gene polymorphisms as risk factors for breast cancer: an Italian case-control study. BMC cancer. 2009, 9:115

53. Hosseini M, Houshmand M, Ebrahimi A. Breast cancer risk not only was not associated with CYP17/A2 allele but also was related to A1 allele. Arch Med Sci. 2009, 5:103-106.

54. JakubowskaA, Gronwald J, Menkiszak J, Górski B, Huzarski T, Byrski T, Tołoczko-Grabarek A, Gilbert M, Edler L, Zapatka M, Eils R, Lubiński J, Scott RJ, et al. BRCA1-associated breast and ovarian cancer risks in Poland: No association with commonly studied polymorphisms. Breast Cancer Res Treat. 2009; 119:201-211.

55. MARIE-GENICA Consortium on Genetic Susceptibility for Menopausal Hormone Therapy Related Breast Cancer Risk. Postmenopausal estrogen monotherapy-associated breast cancer risk is modified by CYP17A1_-34 $\mathrm{T}>\mathrm{C}$ polymorphism. Breast Cancer Res Treat. 2009; 120:737-744.

56. Kato I, Cichon M, Yee CL, Land S, Korczak JF. African American-preponderant single nucleotide polymorphisms (SNPs) and risk of breast cancer. Cancer Epidemiol. 2009; 33:24-30.

57. Tuzuner BM, Ozturk T, Kisakesen HI, Ilvan S, Zerrin C, Ozturk O, Isbir T. CYP17 (T-34C) and CYP19 (Trp39Arg) polymorphisms and their cooperative effects on breast cancer susceptibility. In vivo (Athens, Greece). 2010; 24:71-74.

58. Syamala VS, Syamala V, Sheeja VR, Kuttan R, Balakrishnan R, Ankathil R. Possible risk modification by polymorphisms of estrogen metabolizing genes in familial breast cancer susceptibility in an Indian population. Cancer Invest. 2010; 28:304-311.

59. Surekha D, Sailaja K, Rao DN, Padma T, Raghunadharao D, Vishnupriya S. Association of a CYP17 Gene Polymorphism with Development of Breast Cancer in India. Asian Pac J Cancer Prev. 2010; 11:1653-1657.

60. Iwasaki M, Hamada GS, Nishimoto IN, Netto MM, Motola J Jr, Laginha FM, Kasuga Y, Yokoyama S, Onuma H, Nishimura H, Kusama R, Kobayashi M, Ishihara J, et al. Dietary isoflavone intake, polymorphisms in the CYP17, CYP19, 17-HSD1, and SHBG genes, and risk of breast 
cancer in case-control studies in Japanese, Japanese Brazilians, and Non-Japanese Brazilians. Nutr and Cancer. 2010; 62:466-475.

61. Kaufman B, Laitman Y, Ziv E, Hamann U, Torres D, Lahad EL, Beeri R, Renbaum P, Jakubowska A, Lubinski J, Huzarski T, Toloczko-Grabarek A, Jaworska K, et al. The CYP17A1 -34T > C polymorphism and breast cancer risk in BRCA1 and BRCA2 mutation carriers. Breast Cancer Res Treat. 2011; 126:521-527.

62. Ghisari M, Eiberg H, Long M, Bonefeld-Jorgensen EC. Polymorphisms in phase I and phase II genes and breast cancer risk and relations to persistent organic pollutant exposure: a casecontrol study in Inuit women. Environ Health. 2014; 13:19.
63. Chattopadhyay S, Siddiqui S, Akhtar MS, Najm MZ, Deo SV, Shukla NK, Husain SA. Genetic polymorphisms of ESR1, ESR2, CYP17A1, and CYP19A1 and the risk of breast cancer: a case control study from North India. Tumor Biology. 2014; 35:4517-4527.

64. Karakus N, Kara N, Ulusoy AN, Ozaslan C, Tural S, Okan I. Evaluation of CYP17A1 and LEP Gene Polymorphisms in Breast Cancer. Oncol Res Treat. 2015; 38:418-422.

65. Farzaneh F, Noghabaei G, Barouti E, Pouresmaili F, Jamshidi J, Fazeli A, Emamalizadeh B, Darvish H. Analysis of CYP17, CYP19 and CYP1A1 Gene Polymorphisms in Iranian Women with Breast Cancer. Asian Pac J Cancer Prev. 2016; 17:23-26. 